\title{
A Fault Diagnosis Scheme for Three Phase Induction Motors based on Uncertainty Bounds
}

\author{
Mohammed Obaid Mustafa, George Nikolakopoulos, Thomas Gustafsson \\ Department of Computer Science, Electrical and Space Engineering \\ Luleå University of Technology \\ Luleå, Sweden
}

\begin{abstract}
The aim of this article is to present a fault diagnosis scheme for the case of squirrel-cage Three Phase Induction Motors based on uncertainty bounds violation conditions. The suggested scheme has the capability to diagnose two types of faults: a) broken rotor bar and b) short circuit in stator winding. The fault diagnosis is being performed through a two steps procedure. In the first step the parameters of the healthy induction motor are being identified by utilizing a Set Membership Identification approach, where corresponding uncertainty bounds are also being provided. In the second step, specific proposed bound violation conditions for the fault detection and fault diagnosis are being on-line evaluated during a sliding time window. Multiple simulation results are being presented that prove the efficacy of the proposed scheme towards fault detection and fault diagnosis.
\end{abstract}

\section{NOMENCLATURE}

$V_{s a}, V_{s b}, V_{s c}:$ Stator's three phase voltages $(V)$

$V_{r a}, V_{r b}, V_{r c}$ : Rotor's three phase voltages $(V)$

$i_{r a}, i_{r b}, i_{r c}$ : Rotor's three phase currents $(A)$

$i_{s a}, i_{s b}, i_{s c}:$ Stator's three phase currents $(A)$

$r_{s}, r_{r}$ : Resistance of stator's and rotor's winding $(\mathrm{Ohm})$

mj $L_{s s}, L_{r r}$ : Stator's and rotor's self-inductances (Henry)

$L_{s}, L_{r}$ : Stator's and rotor's self inductance (Henry)

$L_{m}$ : Mutual inductance (Henry)

$\omega_{r}$ : Rotor's angular speed $(\mathrm{rad} / \mathrm{sec})$

$\omega_{m}$ : Rotor's speed (mechanical) $(\mathrm{rad} / \mathrm{sec})$

$\omega_{s}$ : Supply angular frequency $(\mathrm{rad} / \mathrm{sec})$

$P$ : No. of poles pairs

$J$ : Moment of inertia $\left(\mathrm{Kg} \cdot \mathrm{m}^{2}\right)$

$T_{L}:$ Load torque $(\mathrm{Nm})$

$T_{e}:$ Electromagnetic torque $(\mathrm{Nm})$

$q$ : Quadrature axis frame

$d$ : Direct axis frame

$s:$ Stator quantities

$r:$ Stator quantities

$\psi_{s}, \psi_{r}$ : Stator's and Rotor's fluxes (Weber)

$\theta$ : Angular position in the frame of motor $(\mathrm{Deg})$

$\theta_{r}$ : Angle between rotor's phase axis and stator's phase axis

$\beta$ : Angle between rotor's phase axis and stator's phase axis

$N_{b}$ : Numbers of machine rotor bars

\section{INTRODUCTION}

Induction motors are essential components in the vast majority of industrial processes, while due to the fact that these motors are operating in difficult working environments, there are numerous degrading factors such as: dust, temperature variations, humidity, continuous operation, and heavy loads, that effect the motor performance and are capable of creating internal faults. Different types of motor faults may result in different types of motor break down and sometimes in a significant impact on the uninterrupted industrial process operation [1].

Among the most common faults that can be found in the area of induction motors are: a) opening or shorting of one or more of a stator's phase winding [2], b) broken rotor bar or cracked rotor's end-rings [3], c) static or dynamic air-gap irregularities [1], and d) bearing failures [4].

For detecting the mechanical or electrical faults in a three phase induction motor, multiple methods have been utilized in the relative literature such as: 1) Artificial Neural Networks [5], 2) Fast Fourier Transforms [6], 3) Time Step Coupled Finite Element-State Space [5], 4) Motor Current Signature Analysis [7], 5) Wavelet, and Complex Park Vectors [5], while all of these methods base their operation on spectral analysis of stator currents [8], stator voltages, and electromagnetic torque [9]. Recently, identification or prediction techniques have been utilized to perform diagnosis procedure for the occurred faults [6].

In parallel to these fault diagnosis schemes, Set Membership Identification (SMI) [10], [11] has received a growing attention in the past years as a quite important technique for system identification with uncertainty bounds [12], [13], [14], [15].

The rest of the article is being structured as it follows. In Section II the model derivation and simplification, for the healthy and the faulty cases is being derived. In Section III the Set Membership Identification scheme is being presented, followed by the proposed fault detection conditioning framework in Section IV. Section V contains multiple simulation results that prove the efficacy of the proposed methodology in fault detection and fault diagnosis, while the conclusions are drawn in the last Section VI.

\section{INDUCTION MOTOR MODELING}

\section{A. Healthy Case}

In general an induction motor can be modeled as a three phase model or as an equivalent quadrature phase model [16], [17], [18], while the three phase model can be further simplified by the utilization of the equivalent two phase model, which has been converted to the $q-d$ coordination frame [19]. Based on this approach, the original $a b c$ variables $F_{a b c}$ should 
be transformed into the corresponding $d-q$ variables $F_{q d o}$ and this is being carried out through the utilization of Park's transform [20]. In the case that the saturation and the fraction effects are being neglected, the state space equations of the three phase induction motor are:

$$
\left[\begin{array}{c}
\frac{d i_{q s}}{d t} \\
\frac{d i_{d s}}{d t} \\
\frac{d i_{q r}}{d t} \\
\frac{d i_{d r}}{d t}
\end{array}\right]=\mathbf{A}\left[\begin{array}{c}
i_{q s} \\
i_{d s} \\
i_{q r} \\
i_{d r}
\end{array}\right]+\mathbf{B}\left[\begin{array}{c}
V_{q s} \\
V_{d s} \\
0 \\
0
\end{array}\right]
$$

where:

$$
\begin{aligned}
& \mathbf{A}=\frac{1}{\delta}\left[\begin{array}{cccc}
-L_{r} r_{s} & 0 & L_{m} r_{s} & 0 \\
0 & -L_{r} r_{s} & 0 & L_{m} r_{s} \\
L_{m} r_{r} & 0 & -L_{s} r_{r} & w_{r} \delta \\
0 & L_{m} r_{r} & -w_{r} \delta & -L_{s} r_{r}
\end{array}\right] \\
& \mathbf{B}=\frac{1}{\delta}\left[\begin{array}{cccc}
L_{r} & 0 & -L_{m} & 0 \\
0 & L_{r} & 0 & -L_{m} \\
-L_{m} & 0 & \bigsqcup_{s} & 0 \\
0 & -L_{m} & 0 & L_{s}
\end{array}\right]
\end{aligned}
$$

and $\delta=L_{s} L_{r}-L m^{2}$.

\section{B. Broken Bar Model of Three Phase Induction Motor}

The event of a broken rotor bar causes asymmetry in the resistance and inductance of the rotor's phases, which results in asymmetry of the rotating electromagnetic field in the air gap between stator and rotor. Consequently, this will induce frequency harmonics in the stator current. The impact of broken rotor bars can be modeled by unbalancing the rotor resistance, while the inductance changes are being neglected due to their insignificance influence compared to the resistance changes [21], [22]. The stator resistances and inductances stay unchanged [22] and for simplicity purposes, for a squirrelcage rotor, the end-ring contribution is being also neglected.

In the case of such a fault, the modified (faulty) version of matrix $\mathbf{A}_{\mathbf{b}}^{*}$ should be utilized. The resistance changes are being derived based on the assumption that the broken bars are contiguous, neither the end ring resistance nor the magnetizing current is taken into account. For the case of $n_{b b}$ broken rotor bars, the increment $\Delta r_{r a, b, c}$ in each phase is being obtained as [22], [23]:

$$
\Delta r_{r a, b, c}=r_{r} \frac{3 n_{b b}}{N_{b}-3 n_{b b}}
$$

For representing mathematically the broken bar fault fault, the rotor resistance matrix needs to be replaced by the modified rotor resistance matrix $r_{r f}$ and transformed to the $q-d$ reference frame, by utilizing the stationary reference frame. For the cased examined, the rotor resistance is being transformed in the $q-d$ reference frame as in [22].

Therefore the state space matrices in the faulty case will become $\mathbf{A}_{\mathbf{b}}^{*}, \mathbf{B}_{\mathbf{b}}^{*}$ and are being defined as:

$$
\mathbf{A}_{\mathbf{b}}^{*}=-\mathbf{R}_{\mathbf{b r}}^{*} \mathbf{L}^{-1}
$$

with

$$
\mathbf{A}_{\mathbf{b}}^{*}=\frac{1}{\delta}\left[\begin{array}{cccc}
-L_{r} r_{s} & 0 & L_{m} r_{s} & 0 \\
0 & -L_{r} r_{s} & 0 & L_{m} r_{s} \\
L_{m} r_{r q} & 0 & -L_{s} r_{r q} & w_{r} \delta \\
0 & L_{m} r_{r d} & -w_{r} \delta & -L_{s} r_{r d}
\end{array}\right]
$$

$$
\mathbf{B}_{\mathbf{b}}^{*}=\mathbf{B}
$$

\section{Stator Winding Short Circuit Modeling}

In the examined case all the stator parameters are considered to be identical when short circuit happens in the winding of the three phase induction motor, while both stator's resistance and inductance, as also the mutual inductances between stator and rotor will be directly affected. In the case of such a fault, the corresponding modified (faulty) state space motor realization should be utilized. In the examined case, the $q-d$ reference frame transformation needs to be performed to these equations and by assuming that the short circuit occurs only in phase $a$ of the stator, for simplification reasons and without loosing generality, the resistance and inductance matrix of the stator, with shorted turns, are being defined in the $q-d$ reference framed as in [22].

\section{ARMA Model Transformatiokn}

The $q-d$ model of the induction motor is being transformed to a MIMO ARMA system that can be provided by:

$$
\left[\begin{array}{c}
i_{q s}(t) \\
\dot{i}_{d s}(t) \\
\dot{i}_{q r}(t) \\
\dot{i}_{d r}(t)
\end{array}\right]=\left[\begin{array}{c}
\theta_{q s}(t) \\
\theta_{d s}(t) \\
\theta_{q r}(t) \\
\theta_{d r}(t)
\end{array}\right]^{T} \cdot\left[\begin{array}{l}
\Phi_{q s}(t) \\
\Phi_{d s}(t) \\
\Phi_{q r}(t) \\
\Phi_{d r}(t)
\end{array}\right]^{T}+\left[\begin{array}{c}
e_{q s}(t) \\
e_{d s}(t) \\
e_{q r}(t) \\
e_{d r}(t)
\end{array}\right]
$$

where $\theta_{j}(t)$ is the parameter vector sets and the subindex $j$ represents the current set that can be selected as one from: $[q s, d s, q r, d r]$. Moreover $\theta_{j}(t)$ contains the corresponding coefficients of the selected ARMA model and can be defined in the general case as:

$$
\theta_{j}^{T}(t)=\left[\begin{array}{llll}
F_{j, 1}(t) & \ldots & F_{n, 1}(t), T_{j, 1}(t), \ldots, T_{m, 1}(t)
\end{array}\right]^{T}
$$

Where $n$ and $m$ are the orders of the numerator and denominator for each considered transfer function respectively. The regression vector $\Phi_{j}(t)$ is being formulated as:

$$
\Phi_{j}^{T}(t)=\left[-y_{j}(t-1), \ldots,-y_{j}(t-n), \ldots, u_{j}(t+m-n-1), \ldots, u_{j}(t-n)\right]
$$

In equation (1) corrupting noise effecting the measurements is also taken under consideration, while it is assumed that this noise sequence is bounded by $\gamma_{j} \in \mathfrak{R}^{+}$as:

$$
\gamma_{j}\left\|e_{j}(t)\right\|^{2} \leq 1, \forall t
$$

In this MIMO ARMA modeling approach, the parameters in equation (1) for the healthy case of $j=q s$ are being defined as:

$$
\begin{aligned}
T_{q s, 1}= & \frac{L_{r}}{\delta} \\
T_{q s, 2}= & b_{1}\left(a_{1}+2 a_{4}+a_{2} b_{2}\right) \\
T_{q s, 3}= & b_{1}\left(a_{4}^{2}-2 a_{1} a_{4}-a_{5}^{2}+a_{2} a_{3}-a_{2} b_{2}\left(a_{1}+a_{4}\right)\right) \\
T_{q s, 4}= & -b_{1}\left(a_{1} a_{4}^{2}-a_{2} * a_{3} * a_{4}+a_{1} * a_{5}^{2}+\right. \\
& \left.a_{2} b_{2}\left(a_{1} a_{4}-a_{2} a_{3}\right)\right] \\
F_{q s, 1}= & -\left(2 a_{1}+2 a_{4}\right) \\
F_{q s, 2}= & a_{1}^{2}+4 a_{1} a_{4}-2 a_{2} a_{3}+a_{4}^{2}+a_{5}^{2} \\
F_{q s, 3}= & -2 a_{1}^{2} a_{4}+2 a_{1} a_{2} a_{3}-2 a_{1} a_{4}^{2} \\
& -2 a_{1} a_{5}^{2}+2 a_{2} a_{3} a_{4} \\
F_{q s, 4}= & a_{1}^{2} a_{4}^{2}+a_{1}^{2} a_{5}^{2}-2 a_{1} a_{2} a_{3} a_{4}+a_{2}^{2} a_{3}^{2}
\end{aligned}
$$


with:

$$
\begin{array}{cc}
a_{1}=-\left(\begin{array}{ll}
L_{r} & r_{s}
\end{array}\right) / \delta & a_{5}=\omega_{r} \\
a_{2}=\left(\begin{array}{ll}
L_{m} & r_{s}
\end{array}\right) / \delta & b_{1}=L_{r} / \delta \\
a_{3}=\left(\begin{array}{ll}
L_{m} & r_{r}
\end{array}\right) / \delta & b_{2}=-L_{m} / \delta a_{4}=-\left(L_{s} r_{r}\right) / \delta
\end{array}
$$

The adopted realization can be straight forward applied for all the examined faulty cases, while due to its simple derivation and space limitations it has been omitted in this article.

\section{Set Membership Fault Identification}

The objective of the SMI technique is the determination of the feasible parameter set that contains the nominal parameter vector and is consistent with a linearly parameterizable model, the measurement data and the a priori known bounded noise-error. Due to the complexity in computing the feasible parameter set, the majority of the SMI methods aims at the determination of a more conveniently computable parametric set that outer bounds the feasible parameter set [24], [10].

The SMI technique is based on the Weighted Recursive Least Squares (WRLS) with a forgetting factor for identifying the $\hat{\theta}_{j}$ motor's parameters and can be formulated by the following double recursions [25] in the sample instance $t$ and for the MIMO case $j$ as:

$$
\begin{aligned}
\hat{\theta}_{j}(t) & =\hat{\theta}_{j}(t-1)+K_{j}(t)\left(y_{j}(t)-\Phi_{j}^{T}(t) \theta_{j}(t-1)\right) \\
K_{j}(t) & =P_{j}(t) \Phi_{j}(t)=P_{j}(t-1) \Phi_{j}(t)\left(\lambda+\Phi_{j}^{T}(t) P_{j}(t-1) \phi_{j}(t)\right)^{-1} \\
P_{j}(t) & =\left(I-K_{j}(t) \Phi_{j}^{T}(t)\right) P_{j}(t-1) / \lambda \\
e_{j}(t) & =y_{j}(t)-\Phi_{j}^{T}(t) \theta_{j}(t-1) \\
G_{j}(t) & =\Phi_{j}^{T}(t) P_{j}(t-1) \Phi(t)
\end{aligned}
$$

In the SMI approach the initial bounds $\gamma$ for the corrupting noise $\varepsilon_{j}(t)$ are being re-calculated in every iteration. This optimization in the uncertainty description is evolving with the time, as the better the knowledge of the parameters is, the smaller these bounds are. To calculate the optimal value of $\lambda_{j}^{*}(t)$ for achieving convergence, the maximum positive root of the following equation should be extracted in each iteration:

$$
\begin{aligned}
F_{j}\left(\lambda_{j}\right) & =\alpha_{2, j} \lambda_{j}^{2}+\alpha_{1, j} \lambda_{j}+\alpha_{0, j} \\
\alpha_{2, j} & =(\ell+n-1) G_{j}^{2} \\
\alpha_{1, j} & \left.=\left(2 \ell+2 n-1+\gamma_{j} e_{j}^{2}\right)-\xi_{j} \gamma_{j} G_{j}\right) G_{j} \\
\alpha_{0, j} & =(\ell+n)\left(1-\gamma_{j} e_{j}^{2}\right)-\xi_{j} G_{j} \gamma_{j} \\
\xi_{j}(t) & =\xi_{j}(t-1)+\frac{\lambda_{j}}{\gamma_{j}}-\frac{\lambda_{j} e_{j}}{1-\lambda_{j} G_{j}}
\end{aligned}
$$

with $\ell=m+1$. For calculating the upper and lower boundary of the identified parameters, the uncertainty bounds $\sigma_{j}(t)$, should be computed in every iteration. These bounds are being defined by an ellipsoid that bounds the uncertainty and it is oriented parallel to the parameter coordinate axes, defined as:

$$
\sigma_{j}(t)=\sqrt{\operatorname{diag}\left(P_{j}(t)\right)}
$$

while the corresponding equation for the ellipsoids $\Omega_{j}^{e}(t)$ can be calculated as [10], [26]:

$$
\left.\Omega_{j}^{e}(t)=\left\{\theta_{j}:\left(\theta_{j}(t)-\hat{\theta}_{j}(t)\right)^{T} \frac{C_{j}(t)}{\xi_{j}(t)}\right)\left(\theta_{j}(t)-\hat{\theta}_{j}(t)\right) \leq 1, j=1, \ldots, n+m\right\}
$$

The covariance matrix is denoted as $C_{j}(t)=P_{j}(t)^{-1}$. The matrix $W(t)=\frac{C_{j}(t)}{\xi_{j}(t)}$ will represent how far the ellipsoid extends in each direction from the center of the ellipsoid $\hat{\theta}_{j}(t)$, while the volume ratio of the $j$ ellipsoid is being calculated by:

$$
B_{j}(t)=\operatorname{det}^{-1}(W(t))
$$

\section{Fault Detection and Diagnosis Conditioning}

In both cases of a fault occurrence due to short circuit in stator winding or rotor broken bar, the values of the identified parameters will be characterized by a jump and a constant drift from the converged nominal values of the healthy motor. This jump has a direct effect on the updated parameter's bounds, as after the event of the fault the following calculated uncertainty bounds will exceed the previous calculated bounds in the healthy case, while this situation is a direct indication of a fault occurrence. Based on the proposed SMI scheme, the following rules for the fault detection and diagnosis will be established as it is being depicted in Figure 1. In the presented approach it has been assumed that the SMI scheme is providing smooth value updates for the identified parameters.

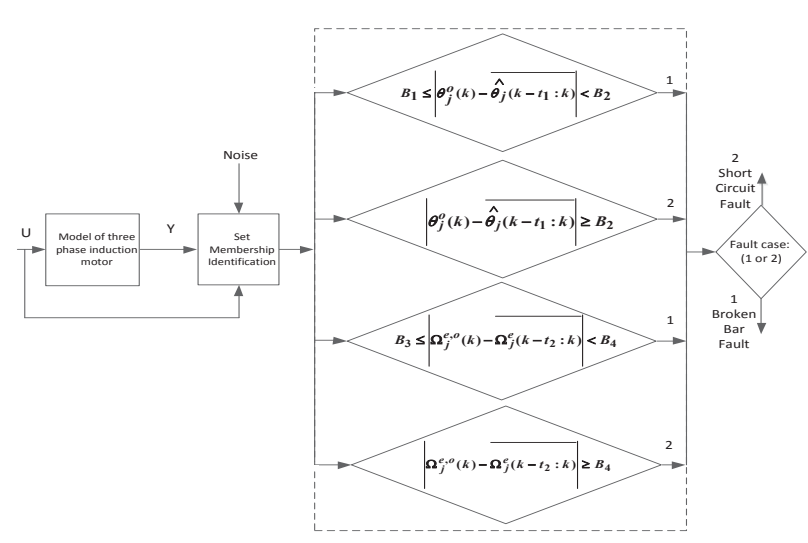

Fig. 1. Conditions for fault Diagnosis

If a $t_{1}$ time window is being defined, then after the convergence of the parameters, small changes in the identified values should be allowed, while a significant change might indicate the occurrence of a fault. For the ad-hoc defined bounds $B_{1}, B_{2}$ the fault diagnosis conditions are being formulated as:

$$
\begin{array}{ll}
{[\underline{\text { Condition } 1}]} & B_{1} \leq \hat{\theta_{j}^{o}(k)-\hat{\theta_{b r, j}\left(k-t_{1}: k\right)}} \mid<B_{2} \\
{[\underline{\text { Condition 2 }}]} & \theta_{j}^{o}(k)-\hat{\theta_{s h, j}\left(k-t_{1}: k\right)} \mid \geq B_{2}
\end{array}
$$

where $\theta_{j}^{o}(k)$ denotes the $j$-converged identified parameter, the notation - represents a moving average time window of length $t_{1}$, while $\theta_{b r, j}$ and $\theta_{s h, j}$ represent the $j$-identified parameter for the cases of a broken bar and a short circuit fault respectively. Additional rules can be defined, that are related with the volume of the bounding ellipsoids $\Omega_{j}^{e}$. The 
aim is to track the corresponding volume of the bounding ellipsoid and allow only small changes, as in the opposite case, this should generate a fault. By defining the ad-hoc boundaries $B_{3}$ and $B_{4}$ over a time window $t_{2}$, the following additional fault diagnosis conditions can be formulated:

$$
\begin{aligned}
& \text { [ Condition 3] } \quad B_{3} \leq\left|\Omega_{j}^{e, o}(k)-\overline{\Omega_{b r, j}^{e}\left(k-t_{2}\right): k}\right|<B_{4} \\
& \text { [Condition 4] } \Omega_{j}^{e, o}(k)-\overline{\Omega_{s h, j}^{e}\left(k-t_{2}\right): k} \mid \geq B_{4}
\end{aligned}
$$

where $\Omega^{e, o}$ is the converged value of the ellipsoid in the healthy case. In the case that conditions (1) and (3) are valid the motor operates under a broken bar fault, while if conditions (2) and (4) are valid then the motor is under a short circuit fault occurrence. Also it should be highlighted that due to this model based fault diagnosis, in the case of a short circuit fault, the fault diagnosis scheme will indicate initially a broken bar fault and while the conditions will continue to be monitored, the fault diagnosis will converge to the correct broken bar diagnosis.

\section{Simulation Results}

The suggested scheme for fault detection and diagnosis is being evaluated on a model of three phase induction motor having the parameters depicted in Table 1.

TABLE I

INDUCTION MOTOR PARAMETERS

\begin{tabular}{|l|c|}
\hline Parameters & Values \\
\hline Pole Numbers & 4 \\
\hline Input Voltage & $240 \mathrm{~V}$ \\
\hline Frequency & $50 \mathrm{~Hz}$ \\
\hline$r_{s}$ & 0.0616 per unit \\
\hline$r_{r}$ & 0.0753 per unit \\
\hline$J$ & $0.00155 \mathrm{Kg} . \mathrm{m} 2$ \\
\hline$N_{b}$ & 28 \\
\hline$L_{s}=L_{r}$ & 0.019 per unit \\
$L_{m}$ & 0.01833 per unit \\
\hline
\end{tabular}

The first simulation results present the effect of $20 \%$ short circuit of stator winding or a one broken rotor bar fault occurrence on the rotor's current, stator's current, torque and angular speed, as it is being presented in Figures 2 and 4 respectively, where the comparison should be made with the healthy case.

As it can be observed from Figure (2), in the event of a fault small increases in the values of the currents $I_{q r}$ and $I_{q s}$ will be induced in both cases. In Figures (3) and (4), it is also depicted that the fault is affecting the steady state values of the rotor's torque and speed respectively. In the case of the motor's torque oscillations are taking place in both types of fault, while the amplitude of these oscillations is being increased with respect to the number of the faulty broken bars and percentage of short circuit in stator winding. Moreover, the speed is also being decreased in the case of broken rotor bar and will be increased in the short circuit case, while oscillations are also evident.

The convergence of the SMI identification for the motor's parameters in the healthy case, from $V_{q s}$ to $i_{q s}$ is being

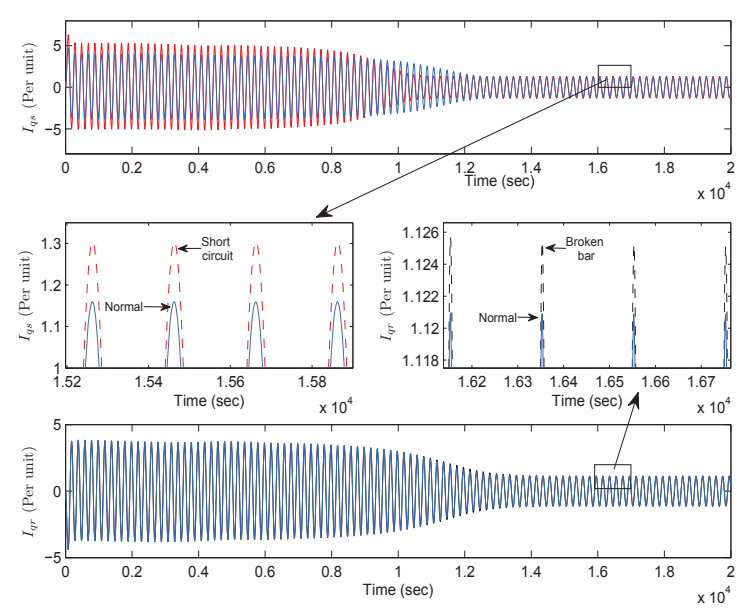

Fig. 2. The rotor's $I_{q r}$ and stator's $I_{q s}$ currents in the normal and faulty cases

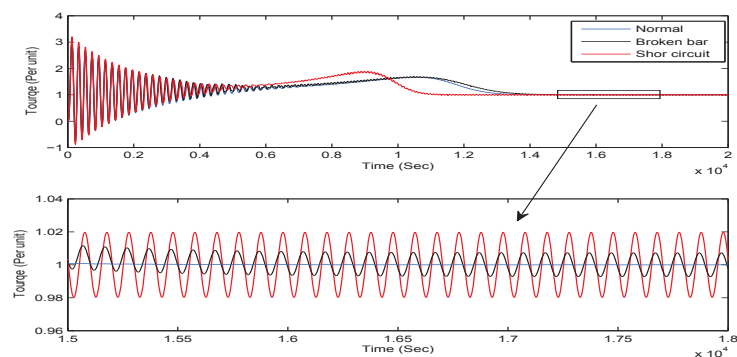

Fig. 3. The motor's torques in the normal and faulty cases

presented in Figures 5 and 6, while similar results have been obtained for the rest of the motor's parameters, but due to space limitations, those graphs have been omitted. As it can be observed from these figures the uncertainty bounds are starting from a large value and in the sequence, as the identification procedure is evolving and the identified parameters are close to the nominal values, those bounds are being decreased, until reach their steady state value. Due to the scale of the presented results, it should be noted that all the parameters have been

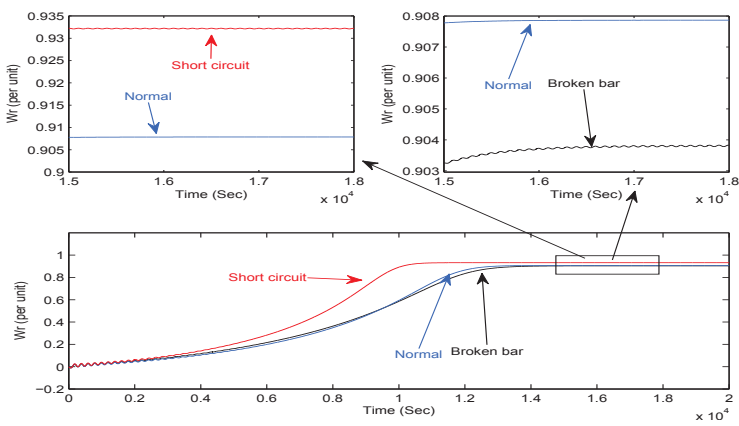

Fig. 4. The rotor's angular speeds in the normal and faulty cases 
converged to their nominal values, as it is also being indicated from the convergence of the ellipsoid volume in Figure 7, for the healthy, broken rotor bar, and short circuit cases. After the event of the fault, the volume is kept decreasing as the examined types of faults are causing a model drift to smaller ARMA parameters than in the healthy case.

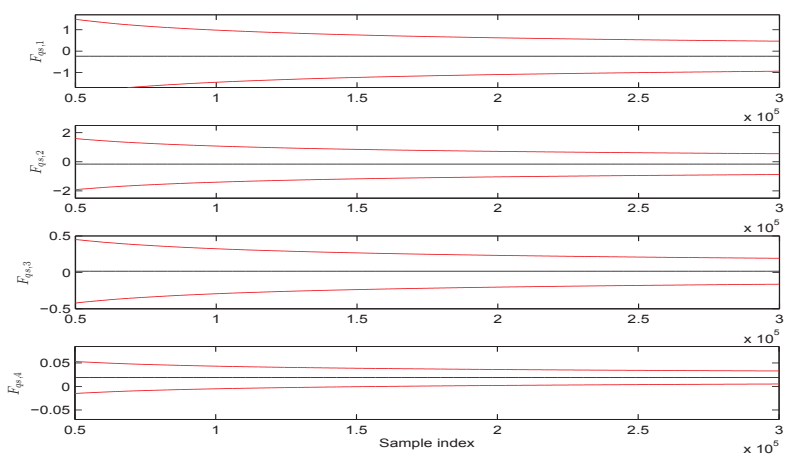

Fig. 5. SMI based identified parameters for $F_{i_{q s}}$ and corresponding uncertainty bounds
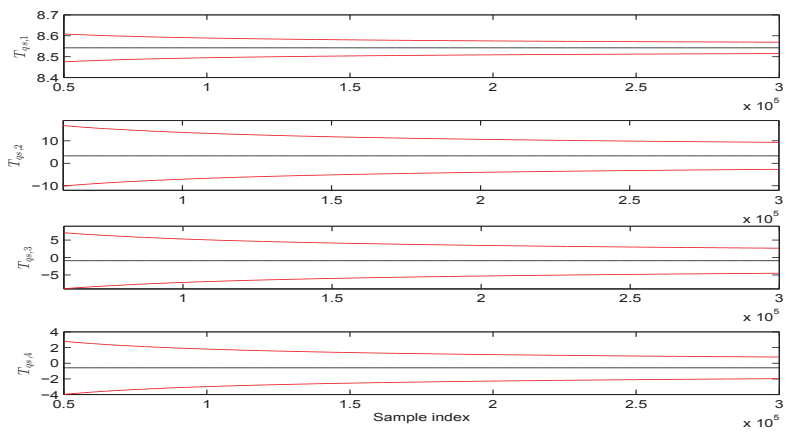

Fig. 6. SMI based identified parameters for $T_{i_{q s}}$ and corresponding uncertainty bounds

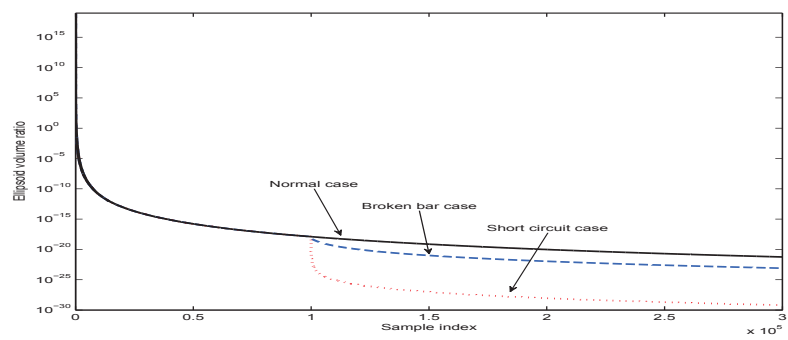

Fig. 7. Ellipsoid volume ratio change with sample index

In Figure 8, the cases of a broken bar or a shot circuit fault occurred at $10^{5}$ samples (sampling time $T_{s}=0.01$ ) are being presented. Due to this fault a jump in the value of the identified parameter is taking place that affects the proposed conditions for fault diagnosis. The uncertainty intervals and the corresponding volumes of ellipsoids are also changing from the nominal converged value (due to the drift in the identified model) and this leads also in different type of bounds violation events, which indicates the existence of a broken bar or short circuit fault. Similar graphs can also be extracted for all the identified parameters of the motor and without loosing generality, in Figures 8, 9 only the results for $T_{q s, 1}, F_{q s, 4}$ are being presented, while in these figures the examined moving time window is also being presented.

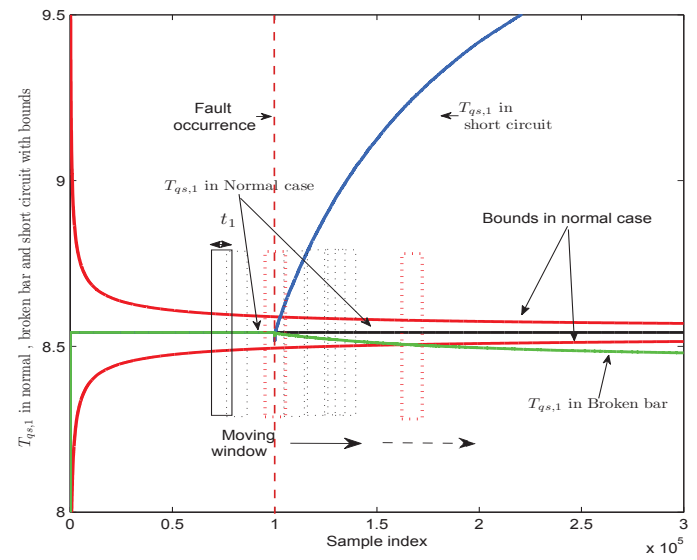

Fig. 8. Convergence of the $T_{q s, 1}$ parameter and corresponding bounds before and after the occurrence of the faults

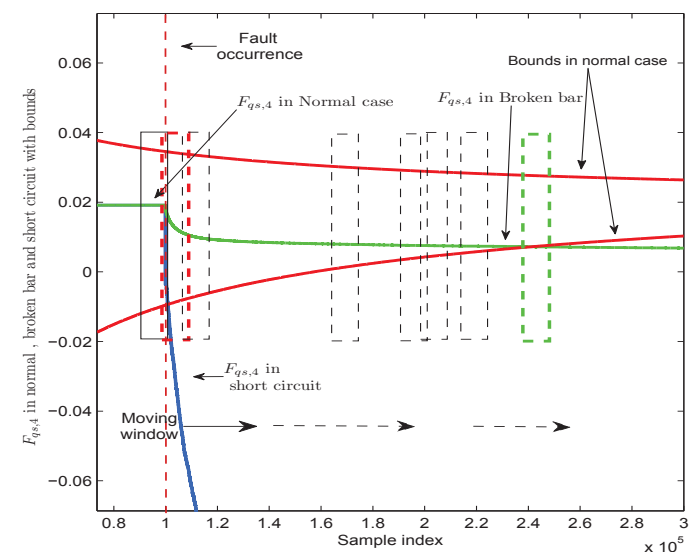

Fig. 9. Convergence of the $F_{q s, 4}$ parameter and corresponding bounds before and after the occurrence of the faults

In Figures 10 and 11 the time evolution of the proposed fault conditions are being depicted in combination with the final fault diagnosis selection, where a fault of type 1 indicates the broken bar fault and a fault of type 2 indicates the short circuit fault. In these figures, the values of the utilized boundaries $\left(B_{1}-B_{2}\right)$ are also depicted.

\section{CONCLUSIONS}

In this article a fault diagnosis scheme for the case of squirrel-cage Three Phase Induction Motors based on uncertainty bounds violation conditions have been presented. 

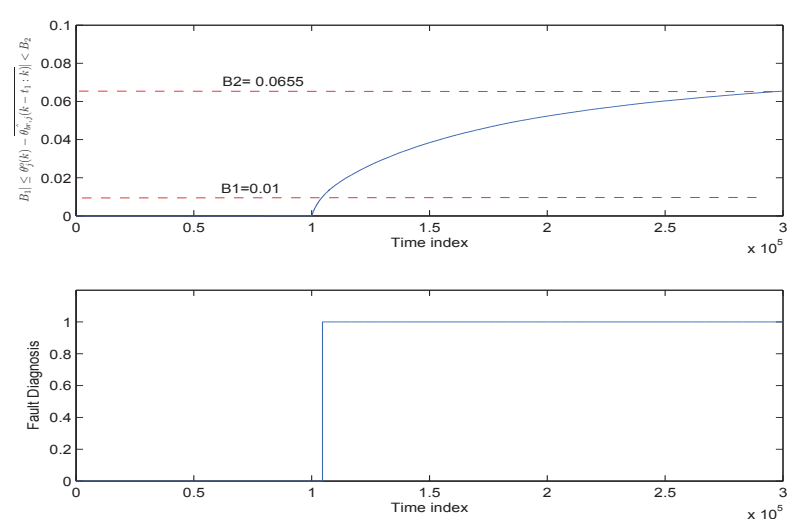

Fig. 10. Broken bar fault diagnosis
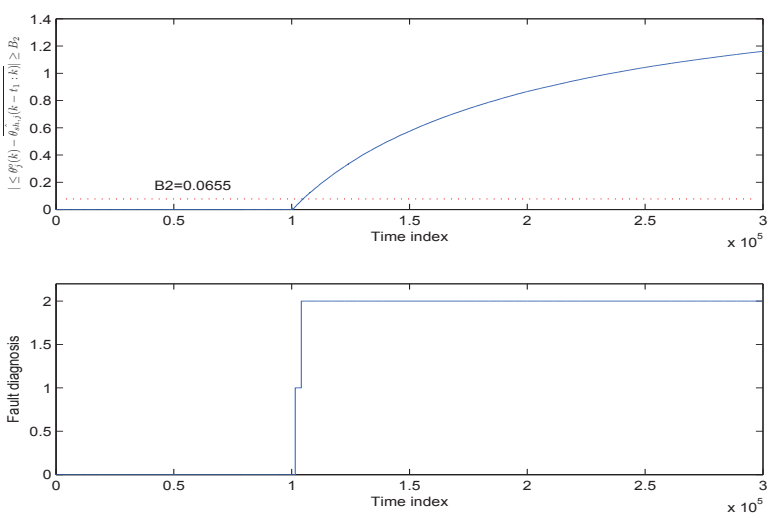

Fig. 11. Short circuit fault diagnosis

The suggested scheme has the capability to diagnose two types of faults: a) broken rotor bar and b) short circuit in stator winding based on a proposed on-line fault condition monitoring scheme. Multiple simulation results have been presented that prove the efficacy of the proposed scheme towards fault detection and fault diagnosis.

\section{REFERENCES}

[1] G. G. Acosta, C. J. Verucchi, and E. R. Gelso, "A current monitoring system for diagnosing electrical failures in induction motors," Mechanical Systems and Signal Processing, vol. 20, no. 4, pp. 953-965, Oct 2004.

[2] S. Nandi and H. Toliyat, "Condition monitoring and fault diagnosis of electrical machines-a review," IEEE Transactions on Energy Conversion, vol. 20, no. 4, pp. 719-729, Dec. 2005.

[3] P. Santos and T. Lubiny, "A simplified induction machine model to study rotor broken bar effects and for detection," European Transactions On Electrical Power, vol. 20, p. 611, 2010.

[4] I. Y. Anel, I. Azenol, and M. E. H. Benbouzid, "Induction motors bearing failures detection and diagnosis using a rbf ann park pattern based method," International Electric Machines and Drives Conference, pp. 1073-1078, Mayl 2007.

[5] K. S. Gaeid and H. A. F. Mohamed, "Bibliography on induction motors faults detection and diagnosis," Australian Journal of Basic and Applied Sciences, vol. 4, no. 2, pp. 227-246, 2010.

[6] S. Bachi, S. Tnani, T. Poinot, and J. Trigeassou, "Stator fault diagnosis in induction machines by parameter estimation," Proc. IEEE Int.SDEMPED, Grado, Italy, p. 235239, Sep. 2001.
[7] I. Aydin, M. Karakose, and E. Aki, "A new method for early fault detection and diagnosis of broken rotor bars," Energy Conversion and Management, vol. 52, no. 4, pp. 1790-1799, April 2011.

[8] H. Douglas, P. Pillay, and A. Ziarani, "Detection of broken rotor bars in induction motors using wavelet analysis," Electric Machines and Drives Conference, 2003. IEMDC'03. IEEE International, pp. 923-928, July 2003.

[9] S. Bachi, S. Tnani, J. Trigeassou, and G. Champenois, "Diagnosis by parameter estimation of stator and rotor faults occurring in induction machines," IEEE Transactions on Industrial Electronics, vol. 53, no. 3, pp. 963-973, June 2006.

[10] J. R. Deller, "Set membership identification in digital signal processing," IEEE ASSP Magazine, vol. 6, no. 4, pp. 4-20, October 1989.

[11] J. Deller, M. Nayeri, and S. Odeh, "Least-Square Identification with Error Bounds for Real-Time Signal Processing and Control," Proceedings of the IEEE, vol. 81, no. 6, pp. 815-849, Jun. 1993.

[12] M.-F. Cheung, S. Yurkovich, and K. Passino, "An optimal volume ellipsoid algorithm for parameter set estimation," IEEE Transactions on Automatic Control, vol. AC-38, no. 8, pp. 1292-1296, Aug. 1993.

[13] G. Belforte, B. Bona, and V. Cerone, "Parameter estimation algorithms for a set-membership description of uncertainty," Automatica, vol. 26, no. 5 , pp. 887-898, 1990.

[14] A. Vicino and G. Zappa, "Sequential approximation of feasible parameter sets for identification with set membership uncertainty," IEEE Transactions on Automatic Control, vol. 41, pp. 774-783, Sep. 1996.

[15] J. Watkins and S. Yurkovich, "Parameter set estimation algorithms for time-varying systems," International Journal of Control, no. 5, pp. 711732, Mar. 1997.

[16] M. Obaid, G. Nikolakopoulos, and T. Guastafsson, "A survey on modeling approaches for three phase induction motors," The IASTED International Conference on Modelling, Simulation, and Identification, MSI 2011, November 2011.

[17] P. Vas, Electrical Machines and Drives, O. S. Publication, Ed., 1992.

[18] A. Sarkar and G. Berg, "Digital simulation of three-phase induction motors," IEEE Transactions on Power Apparatus and Systems, vol. 89, no. 6, pp. 1031-1037, July/August 1970.

[19] K. Sandhu and V. Pahwa, "Sumilation study of three phase induction motor with variation in moment of inertia," ARPN Journal of Enginering and Apllied Sciences, vol. 4, no. 5, pp. 72-77, August 2009.

[20] R. Lee, P. Pillay, and R. Harley, "Dq reference frames for the simulation of induction motors," Electric Power Systems Research, vol. 8, pp. 15 $-26,1985$.

[21] A. Bellini, F. Filippetti, G. Franceschini, C. Tassoni, and G. Kliman, "Quantitative evaluation of induction motor broken bars by means of electrical signature analysis," IEEE Transactions on Industry Application, vol. 37, no. 5, pp. 1248-1255, Sept/Oct 2001

22] S. Chen and R. Zivanovic, "Modelling and simulation of stator and rotor fault conditions in induction machines for testing fault diagnostic techniques," European Transactions On Electrical Power, vol. 20, pp. $611-629$, April 2009

[23] S. Kumar, J. Prakash, and S. S. Kumar, "Detection of broken rotor bars in induction motor using derivative free kalman filters," International Conference on Process Automation, Control and Computing (PACC), 2011, pp. 1 - 7, July 2011.

[24] L. Ljung, System Identification Theory For The User. Englewood Cliffs, NJ: Prentice-Hall, 1987.

[25] F. Guastafsson, Adaptive Filtering and Change Detection, J. Wiley and Son, Eds., Sep.2001

[26] K. Le, Z. Huang, C. Moon, and A. Tzes, "Fault detection based on orthotopic set membership identification for robot manipulators," Proceedings of the 17th World Congress The International Federation of Automatic Control, Seoul, Korea,2008, pp. 7344-7349, July 2008. 